\title{
Comparative Analysis of Packet and Trigger Waves Originating from a Finite Wavelength Instability
}

\author{
Vladimir K. Vanag*, ${ }^{\dagger}$ and Irving R. Epstein \\ Department of Chemistry and Volen Center for Complex Systems, MS 015, \\ Brandeis University, Waltham, Massachusetts 02454-9110
}

Received: May 7, 2002; In Final Form: September 19, 2002

\begin{abstract}
The finite wavelength instability generates trigger and packet waves in an extended three-variable Brusselatortype model. The trigger waves account for experimentally observed stacking (shock) structures and acceleration of oncoming waves before collision observed in the Belousov-Zhabotinsky system. Packet waves exhibit specular reflection from surfaces. The mouth of a narrow tube connected to a broader region acts as a semitransparent mirror for packet waves leaving the tube, with a coefficient of transparency that depends on the tube radius.
\end{abstract}

\section{Introduction}

Recent discoveries of antispirals, standing waves, accelerating waves, and packet waves in the Belousov-Zhabotinsky (BZ) system dispersed in water droplets of water-in-oil AOT microemulsions $^{1-3}$ (BZ-AOT system) attract attention to the properties of waves arising from the finite wavelength instability, for which the linearized equations possess a complex conjugate pair of eigenvalues with a positive real part at a characteristic nonzero wavenumber $k_{0}$. Unlike the Hopf instability, $\operatorname{Re}(\lambda)$ may be negative at zero wavenumber $(k=0)$, in which case the homogeneous steady state is stable.

We have shown earlier ${ }^{3}$ that the finite wavelength instability with a negative derivative $\mathrm{d} \omega / \mathrm{d} k$ at $k=k_{0}$ gives rise to antispirals. The finite wavelength instability can also produce standing waves ${ }^{4}$ and packet waves, ${ }^{3}$ which are composed of phase waves. However, it is not clear whether it is possible to obtain trigger waves from a pure finite wavelength instability ("pure" in this case implies that $\operatorname{Re}(\lambda)<0$ at $k=0$, so there is no Hopf instability). If trigger waves can also arise in such a situation, what is the fundamental difference between trigger and packet waves?

In this paper we analyze trigger waves produced by a pure finite wavelength instability in a Brusselator-like reactiondiffusion model and compare these simulated results with our experimental results on accelerating waves in the BZ-AOT system, and with Hamik et al.'s experiments ${ }^{5}$ on stacking or shock structures in the aqueous 1,4-cyclohexanedione BZ system. ${ }^{6}$

Although the Brusselator does not precisely mimic the chemistry of the actual BZ reaction, it is dynamically similar and computationally tractable and has been used in numerous studies of chemical oscillators. For example, both oscillatory cluster patterns in a BZ system subject to periodic perturbation ${ }^{7}$ and competition between Turing and Hopf modes in the chlorite-iodide-malonic acid reaction ${ }^{8}$ have been successfully analyzed with the aid of the Brusselator. We employ a modified Brusselator model, in which time scale factors ${ }^{9}$ bring the model closer to the Oregonator model of the BZ reaction, with different

$\dagger$ Permanent address: Center for Photochemistry, Russian Academy of Sciences, Novatorov str., 7A, 117421, Moscow, Russia. time scales for the activator and the inhibitor, and with an additional variable, a fast diffusing unreactive form of the activator. ${ }^{10-12} \mathrm{We}$ also demonstrate new reflection properties of packet waves and a dependence of their behavior on the radius of a thin tube from which packet waves enter a broader region.

\section{Model and Methods}

Our model starts from the standard Brusselator, ${ }^{13}$ with $u$ as the activator and $v$ as the inhibitor. We supplement this model of the aqueous phase chemistry with two additional features: (a) a time-scale factor $\epsilon_{1} \ll 1$, which allows the Brusselator model to sustain traveling waves $;{ }^{9}$ and (b) a third, rapidly diffusing variable $w\left(D_{w} \gg D_{v}=D_{u}\right)$. The new variable $w$ represents an unreactive form of $u$, analogous to $\mathrm{BrO}_{2}{ }^{\bullet}$ in the oil phase of the BZ-AOT system. The term "unreactive" means that species $w$ does not take part in any chemical reactions in the oil phase other than interconversion with $u$ in the aqueous phase, where it can react with other water-soluble molecules. Species $w$ diffuses more rapidly, because it diffuses as single molecules in the oil phase, whereas $u$ and $v$ diffuse as entire water droplets in water-in-oil microemulsions. ${ }^{1-3}$ The only "reactions" of $w$ are exchange between the two phases, characterized by rate constants $c$ and $d$ and by an additional time-scale factor $\epsilon_{2}$. The resulting system is described by eqs $1-3$, and the unmodified Brusselator is given by eqs 1 and 2 with $c=d=0$ and $\epsilon_{1}=1$. It has been shown previously that both a modified three-variable Brusselator model ${ }^{10,11}$ and model eqs $1-3^{12}$ with $\epsilon_{1}=\epsilon_{2}=1$ generate a finite wavelength

$$
\begin{gathered}
\mathrm{d} u / \mathrm{d} t=\left(a-(1+b) u+u^{2} v-c u+d w\right) / \epsilon_{1}+D_{u} \Delta u \\
\mathrm{~d} v / \mathrm{d} t=b u-u^{2} v+D_{v} \Delta v \\
\mathrm{~d} w / \mathrm{d} t=(c u-d w) / \epsilon_{2}+D_{w} \Delta w
\end{gathered}
$$

instability. Combining all these properties, we have found a set of parameters that produces a pure finite wavelength instability supporting either trigger or packet waves with the same parameters $a, b, c, d$ and $\epsilon_{1}$, but different $\epsilon_{2}$. Dispersion curves (Figure 1) obtained by linear stability analysis demonstrate that the major difference between the two cases is that $\max (\operatorname{Re}(\lambda))$ 


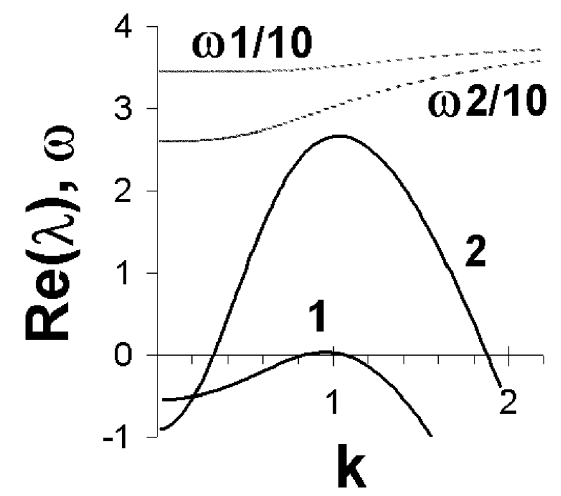

Figure 1. Dispersion curves for system (1) $-(3)$ at $\epsilon_{2}=(1) 0.61$ and (2) 0.2 . Solid curves are $\operatorname{Re}(\lambda)$; dotted lines " $\omega 1 / 10$ " and " $\omega 2 / 10$ " are one-tenth of the imaginary parts $\omega$ of eigenvalues $\lambda$ for cases (1) and (2), respectively. Parameters: $a=3.1, b=3.2, c=2, d=1.5, \epsilon_{1}=$ $0.02, D_{u}=D_{v}=1, D_{w}=20$.

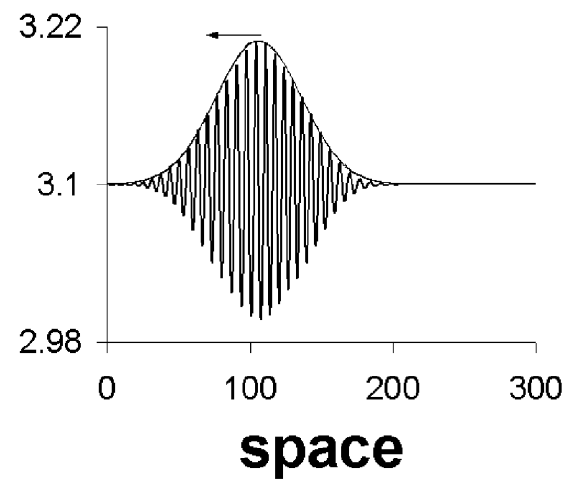

Figure 2. Gaussian wave packet (recorded for variable $u$ ) at $t=100$ with initial perturbation at $t=0: u=u_{\mathrm{SS}}+0.2$ for $299<x<300$. All parameters are as in Figure $1, \epsilon_{2}=0.61$. The arrow shows the direction of the wave packet's propagation. The Gaussian envelope $u_{\mathrm{SS}}$ $+A_{0} /\left(\sigma(2 \pi)^{1 / 2}\right) \exp \left(-\left(x-x_{0}\right)^{2} /\left(2 \sigma^{2}\right)\right)$ has parameters $x_{0}=106, \sigma=$ 30, and $A_{0}=8.2$.

for trigger waves $(\cong 2.67)$ is much greater than the corresponding value $(\cong 0.038)$ for packet waves.

We integrated the partial differential equations (1)-(3) using FlexPDE, ${ }^{14}$ in which a Newton-Raphson iteration process is used with a variable time step and mesh. FlexPDE refines the triangular finite element mesh until the estimated error in any variable is less than a specified tolerance, which we chose as $10^{-4}$, at every cell of the mesh. Calculations were performed with different geometrical shapes of a working area. The initial conditions are the homogeneous steady-state $u_{\mathrm{SS}}=a, v_{\mathrm{SS}}=$ $b / a$, and $w_{\mathrm{SS}}=c a / d$ plus some chosen small local perturbation. Zero-flux boundary conditions are used.

\section{1-D Simulation. Packet Waves}

First, we examine the behavior of system (1)-(3) for $\epsilon_{2}=$ 0.61 (when packet waves are obtained) in one spatial dimension (Figure 2). The homogeneous steady state was initially (at $t=$ 0 ) perturbed at the right boundary. The perturbation grew into a Gaussian wave packet (see also the Gaussian envelope in Figure $4 b$, which fits a wave packet over several orders of magnitude from $10^{-8}$ to nearly $10^{-4}$ ) moving to the left with group velocity $V_{\mathrm{gr}}=1.93$ (measured as the displacement of the maximum amplitude of the packet). The group velocity is nearly equal to $\mathrm{d} \omega / \mathrm{d} k(=2.00)$ at $k=k_{0}(=0.955)$. In simulations of packet waves with other parameters, we have found that the simulated group velocity is always very close to the calculated $\mathrm{d} \omega / \mathrm{d} k$ at $k=k_{0}$. The phase velocity, $V_{\mathrm{p}}$, of individual waves in

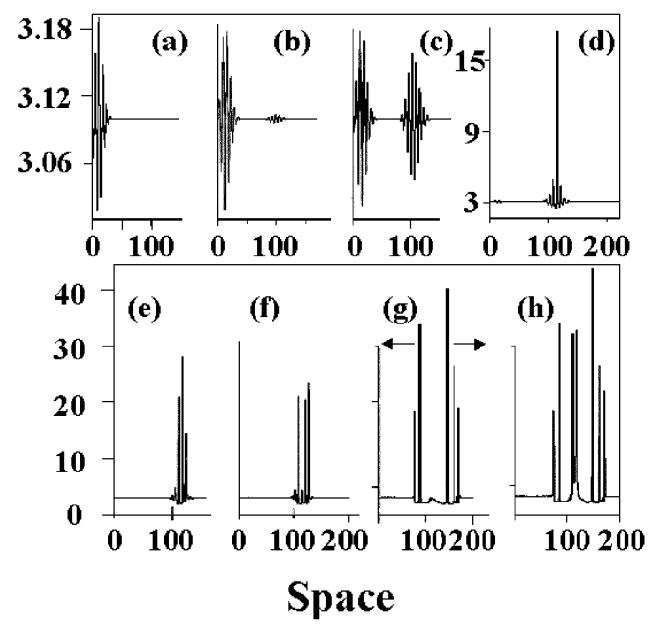

Figure 3. Transformation of packet waves into trigger waves (recorded for variable $u$ ). All parameters are as in Figure 1, $\epsilon_{2}=0.61$ for $0<x$ $<L_{\mathrm{t}}$, and $\epsilon_{2}=0.2$ for $L_{\mathrm{t}} \leq x<400 ; L_{\mathrm{t}}=50 ; t=$ (a) 5.6, (b) 7.0, (c) 8.0 , (d) 9.0 , (e) 9.1 , (f) 9.2 , (g) 10.4, (h)10.46. Initial small perturbation at $t=0$ and $x=0$.

a packet is given by $\omega_{0} / k_{0}$, where $\omega_{0}$ is $\operatorname{Im}(\lambda)$ at $k=k_{0}$. In the present case, $V_{\mathrm{p}}=36.67$. Upon collision, two Gaussian wave packets pass through each other ${ }^{4,12}$ without annihilation, in contrast to the behavior of trigger waves.

In general, the shape of the wave packet depends on both the real and the imaginary parts of the eigenvalue $\lambda(k)$, i.e., on the dispersion curve. Wave packets with nearly square envelopes were also obtained at other system parameters. The behavior of Gaussian wave packets is well-known in optics and quantum mechanics. ${ }^{15-17} \mathrm{~A}$ wave packet propagates with a group velocity, $v_{\mathrm{gr}}=\partial \omega / \partial k$ at $k=k_{0}$, and spreads in time in such a way that the width, $\sigma$, of the Gaussian envelope $\left[\exp \left(-\left(x-x_{0}\right)^{2} / 2 \sigma^{2}\right)\right]$ grows in time as $\sigma=\sigma_{0}\left(1+(t / \tau)^{2}\right)^{1 / 2}$, where $\tau$ is a constant, and $\sigma_{0}=\sigma$ at time $t=0$.

\section{1-D Simulation. From Packet to Trigger Waves}

Trigger waves are obtained at $\epsilon_{2}=0.2$. We used two types of initial perturbation. In this section we describe a soft perturbation. A segment of length $L$ is divided into two zones with $\epsilon_{2}=0.61$ for $0<x<L_{\mathrm{t}}$ and $\epsilon_{2}=0.2$ for $L_{\mathrm{t}}<x<L$. All other parameters and the steady state are the same in both zones. The segment is perturbed at $x=0$. After the perturbation, a packet of waves emerges at the left border and moves to the right (Figure 3a). After some time, a new packet of waves becomes visible in the right zone with $\epsilon_{2}=0.2$ (Figure $3 b$ ). The amplitude of the waves in the right packet grows rapidly (Figures 3b,c) and exponentially (Figure 4a) with an exponent $(\cong 2.7)$ close to $\max (\operatorname{Re}(\lambda))=2.67$. The packet moves with group velocity $V_{\text {gr }} \cong 7.1$, approximately equal to $\mathrm{d} \omega / \mathrm{d} k=7.15$ at $k=k_{0}$.

At about $t=9$ (Figure 3d), the growth rate of the largest peak exceeds the exponential growth rate (see Figure 4a), and the amplitude of the middle wave in the packet reaches a large value around $30-40$ (the steady-state $u_{\mathrm{SS}}=3.1$ ). This is the moment of transition (analogous to a first-order equilibrium phase transition) from packet waves to trigger waves. Immediately after this (Figure 3e,f), the amplitudes of other waves in the packet also reach large values, and a competition among the trigger waves begins. Waves in the center of the packet die, and waves closer to the ends of the packet survive (Figure 3f,g). Due to the oscillatory character of the instability at $k=k_{0}$, new waves emerge (Figure $3 \mathrm{~h}$ ), and a new wavelength is established. 

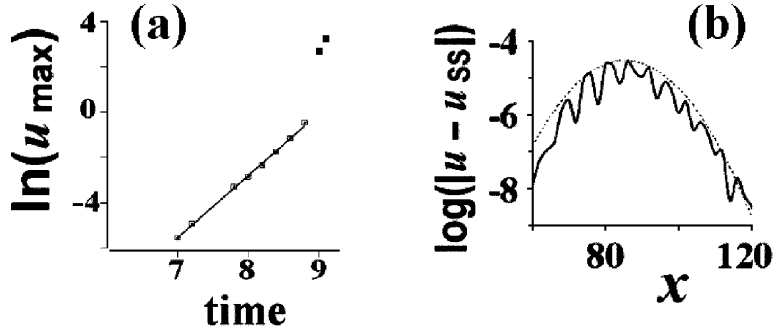

Figure 4. (a) Dependence of maximum amplitude $(u)$ of wave packet on time (squares, solid line is trend line) in semilogarithmic coordinates. (b) Wave packet at $t=5$ in semilogarithmic coordinates. The dotted line is the Gaussian envelope $A_{0} /\left(\sigma(2 \pi)^{1 / 2}\right) \exp \left(-\left(x-x_{0}\right)^{2} /\left(2 \sigma^{2}\right)\right)$ with $x_{0}=L_{\mathrm{t}}+v_{\mathrm{gr}} t, \sigma=7.8$, and $A_{0}=0.000$ 57. All parameters are as in Figure 3 .

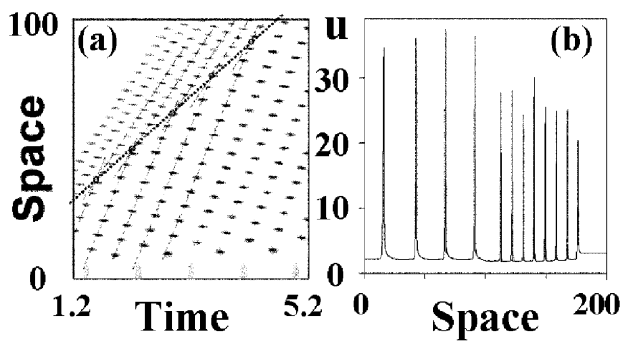

Figure 5. (a) Time - space plot of trigger waves $(u)$ at $\epsilon_{2}=0.2$. Data were accumulated with time interval 0.1 ; movement of the shock point is shown by the bold dotted line. (b) Two groups of trigger waves at $t=5$.1. Initial perturbation of $u_{\mathrm{SS}}$ at $t=0$ and $x=0, u_{0}=15$.

Finally, we obtain two sets of trigger waves moving in opposite directions. The properties of these trigger waves are examined in sections 5-7.

Here we focus on the emergence of the second wave packet in the right zone, at $x>L_{\mathrm{t}}$. Backward extrapolation from the position and velocity of the packet at later times suggests that this wave packet emerges at $t \cong 0$ and $x=L_{\mathrm{t}}$ with an extremely small amplitude. Significant amplification confirms the existence of this very small amplitude wave packet in the earliest stages (Figure 4b). Variation of $L_{\mathrm{t}}$ between 10 and 200 (the wavelength of the packet waves is $2 \pi / k_{0} \cong 6.1$ ) does not reveal any dependence of the time of packet emergence on $L_{\mathrm{t}}$. In all cases, the wave packet emerged at $t \cong 0$. Thus, its initiation has no relation to the phase or group velocity of the left wave packet. Presumably, its emergence at $t \cong 0$ and $x=L_{\mathrm{t}}$ originates from an "infinite" rate of spreading of the initial perturbation due to diffusion. Simulations of system (1)-(3) with $a=b=0$ and with the initial perturbation $u_{0}=1$ at $0<x<1$ and $u_{0}=0$ elsewhere reveal that the spatial distribution of $u$ at very early times ( $t=0.02$, for example) can be described by a Gaussianlike function. For this reason, $u$ exceeds the steady-state value $u_{\mathrm{SS}}$ everywhere, including at $x=L_{\mathrm{t}}$ for $t \cong 0$, leading to the essentially instantaneous emergence of the second wave packet in the presence of a finite wavelength instability.

\section{1-D Simulation. Trigger Waves}

To simulate a "strong" perturbation, we used a homogeneous segment with $\epsilon_{2}=0.2$ and let $u_{0}=15$ (close to the amplitude of the developed trigger waves shown in Figure $3 e-h$ ) for $0<$ $x<1$ and $t=0$. In this case, trigger waves emerge without intermediate packet waves and start to move to the right immediately. New waves periodically emerge at $x=0$ with a period $T=2 \pi / \omega_{0}$, but not all waves survive. Typical wave patterns are presented in Figure 5. Two groups of waves with different wavelength, $\lambda$, velocity, $V$, and amplitude, $A$, are seen in Figure 5b. For the leading group (at the right, group 1), $\lambda_{1} \cong$ 9.3, $V_{1} \cong 34.6$, and $A_{1} \cong 25$, and for the second group, $\lambda_{2} \cong$ $24, V_{2} \cong 54$, and $A_{2}=35-40$. Note that $\omega_{0} / k_{0}=29.5$ and $\lambda=$ $2 \pi / k_{0}=6.1$. Thus, the velocity of the trigger waves is larger than the phase velocity $\omega_{0} / k_{0}$, and the wavelength exceeds $2 \pi /$ $k_{0}$. "Fast" waves from the second group are transformed into "slow" waves of the leading group at the boundary between the first and the second groups of waves, the "shock point". 5

Hamik at al. ${ }^{5}$ observed analogous behavior, which they called (after Howard and Kopell ${ }^{18}$ ) stacking or shock structures, in the $\mathrm{BZ}$ reaction with 1,4-cyclohexanedione as substrate. Until now, there have been no reaction-diffusion models that generate shock structures in autonomous systems. Horikawa has found shock structures in the Fitzhugh-Nagumo model periodically perturbed with external impulses. ${ }^{19}$ The velocity of the shock point may be expressed by the following simple kinematic relation: ${ }^{18,20}$

$$
V_{\text {shock }}=\left(V_{1} \lambda_{2}-V_{2} \lambda_{1}\right) /\left(\lambda_{2}-\lambda_{1}\right)
$$

which yields $V_{\text {shock }} \cong 21.2$ in our case. Direct calculation (dotted line in Figure $5 \mathrm{a}$ ) gives $V_{\text {shock }} \cong 21$.

The stacking phenomenon (often seen in traffic jams) is associated with a negative dispersion $\mathrm{d} V / \mathrm{d} \lambda$, the dependence of the wave velocity on wavelength. ${ }^{21,22}$ Note that $\mathrm{d} V / \mathrm{d} \lambda$ reflects nonlinear interactions between two or more consecutive waves, whereas the dispersion $\mathrm{d} \omega / \mathrm{d} k$ obtained from linear stability analysis reflects only the properties of the steady state.

Like the usual trigger waves found in two-variable models, ${ }^{10,23,24}$ such as the Fitzhugh-Nagumo ${ }^{25}$ or the two-variable Oregonator, ${ }^{26}$ our trigger waves annihilate upon collision with one another or with the zero-flux boundary. This feature (among others) distinguishes them from packet waves, which pass through each other or reflect from the boundary.

\section{2-D Simulation. Accelerating Trigger Waves}

In the previous section we saw how trigger waves can slow if a group of "slow" waves propagates in front of them. The opposite phenomenon can occur when individual trigger waves collide. We observed this behavior in experiments on the BZAOT system at large water droplet concentrations (above the percolation threshold) and interpreted it as resulting from an increase in the wave velocity with decreasing inhibitor concentration. ${ }^{1}$ Later, we suggested that this behavior might arise from acceleration of phase waves in a packet under the geometric constraints of waves entering the region between two wave basins. ${ }^{3}$ This latter argument, however, does not explain several experiments in which we saw wave acceleration before collision in the absence of special geometrical conditions.

Figure 6 shows accelerating trigger waves in model (1)-(3). This behavior resembles that observed in experiments with the BZ-AOT system. Because we have never seen acceleration of approaching trigger waves in two-variable activator-inhibitor models, we conclude that this phenomenon is connected with the third variable, the fast diffusing unreactive form of the activator. Fast diffusion of $w$ leads to depletion of the inhibitor $v$ between two oncoming waves, so the present results support our earlier interpretation.

\section{2-D Simulation. Spirals and Critical Radius for Trigger Waves}

It is well-known ${ }^{27}$ that spirals emerge in an excitable (or oscillatory) medium as the result of a trigger wave break. Open ends of a broken wave evolve due to the dependence of the 
(a)

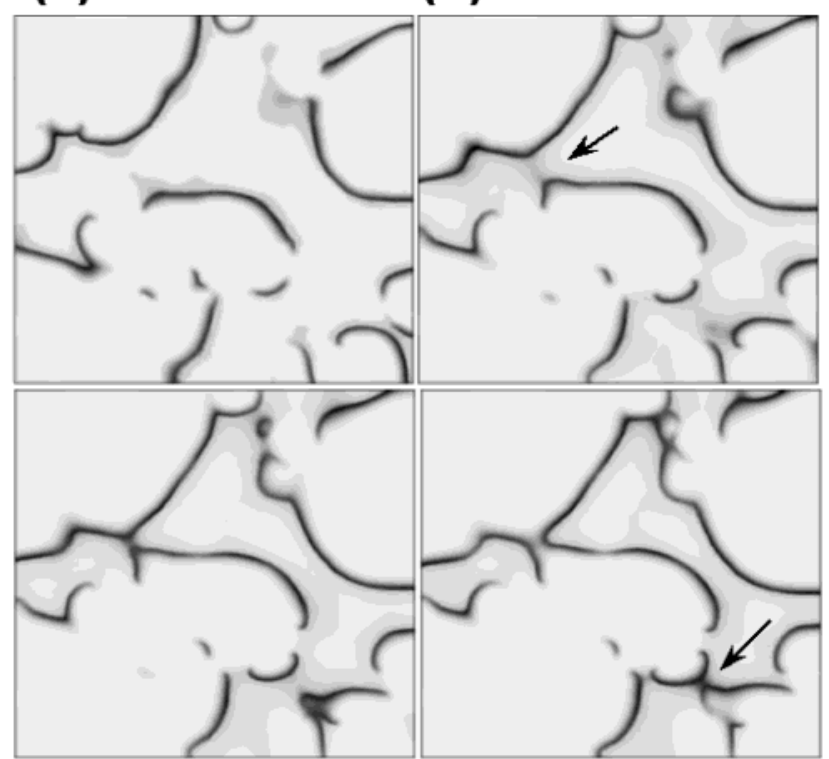

\section{(c)}

(d)

Figure 6. Acceleration of trigger waves before collision. Parameters are as in Figure $1\left(\epsilon_{2}=0.2\right)$, except $a=2.9$, which gives slightly positive $\operatorname{Re}(\lambda)$ at $k=0$ (the homogeneous steady state is unstable to temporal oscillation). Random initial conditions, size $=30 \times 30, t=$ (a) 1.126, (b) 1.146, (c) 1.156, (d) 1.166. Arrows show locations where acceleration of two oncoming waves occurs. Zero-flux boundary conditions.

normal wave velocity, $V(r)$, on the curvature, $1 / r$, according to the relation ${ }^{28-30}$

$$
V(r)=c_{0}-h D / r
$$

where $c_{0}$, which is unique for a given medium, is the velocity of a plane wave, $D$ is the diffusion coefficient of the activator, and $h$ is a constant $\cong 2$. We have already seen that when there is a finite wavelength instability, trigger waves in one spatial dimension (analogue of plane waves) may have two velocities. In addition, we have two forms of the activator with quite different diffusion coefficients $\left(D_{u}\right.$ and $\left.D_{w}\right)$. What are the appropriate values of $D$ and $c_{0}$ in eq 5 ?

First, we wanted to be sure that "normal", outwardly rotating spirals (inwardly rotating "antispirals" are obtained from packet waves $^{2,3}$ ) can be obtained for the case of finite wavelength instability. We used typical initial conditions for spirals in an excitable medium: a broken wave, i.e., two neighboring narrow segments with increased concentration of activator and decreased concentration of inhibitor, with concentrations set at their steadystate values elsewhere. Figure $7 \mathrm{~b}$ shows the spiral that developed at $t=1.2$. The wavelength $\lambda_{\mathrm{S}}$, velocity $V_{\mathrm{S}}$, and amplitude $A_{\mathrm{S}}$ of the spiral wave are 13 (between $\lambda_{1}$ and $\lambda_{2}$ in the onedimensional simulations), 35 (very close to $V_{1}$ ), and 30-35 (between $A_{1}$ and $A_{2}$ ), respectively. Because the curvature of the outermost spiral waves (roughly the reciprocal of the distance from a wave to the center of the spiral) is small $\left(h D / r \ll c_{0}\right.$, even for $\left.D=D_{w}=20\right)$, we conclude that $c_{0} \cong V_{1}$. Our working area (radius $R=30$ ) is not sufficiently large to resolve shock structures, if they exist at all for spiral waves.

If normal spiral waves exist, then eq 5 predicts that at some critical radius $r_{\mathrm{cr}} \cong D / c_{0}$ (for $h=1$ ), waves in a tube of radius $\leq r_{\text {cr }}$ cannot significantly penetrate into a wider region connected to the tube. This prediction was confirmed experimentally in (a)

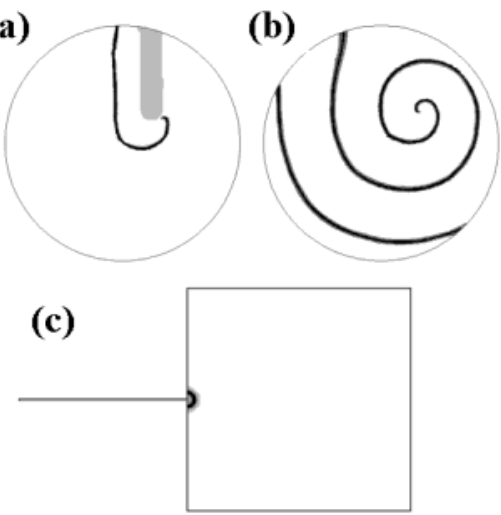

Figure 7. (a, b) Spiral trigger waves. All parameters are as in Figure $1\left(\epsilon_{2}=0.2\right)$. Radius of circle $=30, t=$ (a) 0.2 , (b) 1.2 . The gray stripe in (a) marks the position of the initial decreased concentration of inhibitor $v$, and black thin lines show the activator concentration. (c) Determination of the critical radius for trigger wave transmission. Length of tube $L$ is 15 , and variable radius $r$ of tube is 0.071 for (c); the square is $20 \times 20$. Zero-flux boundary conditions.

the $\mathrm{BZ}$ reaction. ${ }^{31,32} \mathrm{We}$ now examine in a computer experiment its validity for a system with a finite wavelength instability.

In Figure 7c, we show the geometry of our computer experiment, in which a narrow tube is connected to a wider region. A tube of length $L$ and radius $r$ is connected to a large $(20 \times 20)$ square. A trigger wave is excited at the left end of the tube. Depending on the radius $r$, waves can or cannot enter the square. When $r<r_{\text {cr }}$, the wave disappears near the right end of the tube. Figure $7 \mathrm{c}$ shows a case when trigger wave almost enters the square but finally dies $(r=0.071)$. If $r>r_{\mathrm{cr}}$, trigger waves enter the square and propagate as hemispherical waves. We find that $r_{\mathrm{cr}}=0.078$. From eq $5, h D=V_{1} r_{\mathrm{cr}}=2.7$. If $h$ is about 2 , then $D$ is close to $D_{u}=1$, rather than to $D_{w}=$ 20 .

\section{2-D Simulation. Packet Waves. Reflection}

The experimental discovery of antispirals, which are probably a special case of packet waves, ${ }^{2,3}$ raises the question of the conditions under which spirals or antispirals (if $\mathrm{d} \omega / \mathrm{d} k<0$ at $k$ $=k_{0}$, phase waves in a packet propagate toward the center of perturbation) can occur. We therefore carried out a parallel set of computer experiments under the same initial conditions, but with packet waves $\left(\epsilon_{2}=0.61\right)$. Figure $8 \mathrm{a}-\mathrm{d}$ shows that spiral waves do not emerge in this case. Instead, two wave packets diverge from the initially perturbed region. The left one, which is closer to the region of initially increased activator concentration, has a larger amplitude than the right one, which is closer to the region of initially decreased inhibitor concentration.

Upon reaching the circular boundary, the two wave packets reflect in accordance with the rules of geometrical optics, ${ }^{33}$ i.e., the angle of incidence equals the angle of reflection. To our knowledge, this is the first demonstration of specular reflection in reaction-diffusion systems. Earlier, it was shown ${ }^{34}$ that chemical trigger waves obey Snell's law of refraction but do not exhibit specular reflection. To confirm that specular reflection occurs generally for packet waves, we made further computer experiments with several angles of incidence and geometries (Figure $8 \mathrm{e}-\mathrm{h}$ ). In all cases, reflection was specular.

\section{2-D Simulation. Packet Waves. Critical Radius}

The results presented in Figure 8 show that broken wave edges (large curvature) do not lead to curling of packet waves. Therefore, it is conceivable that eq 5 does not hold for packet 

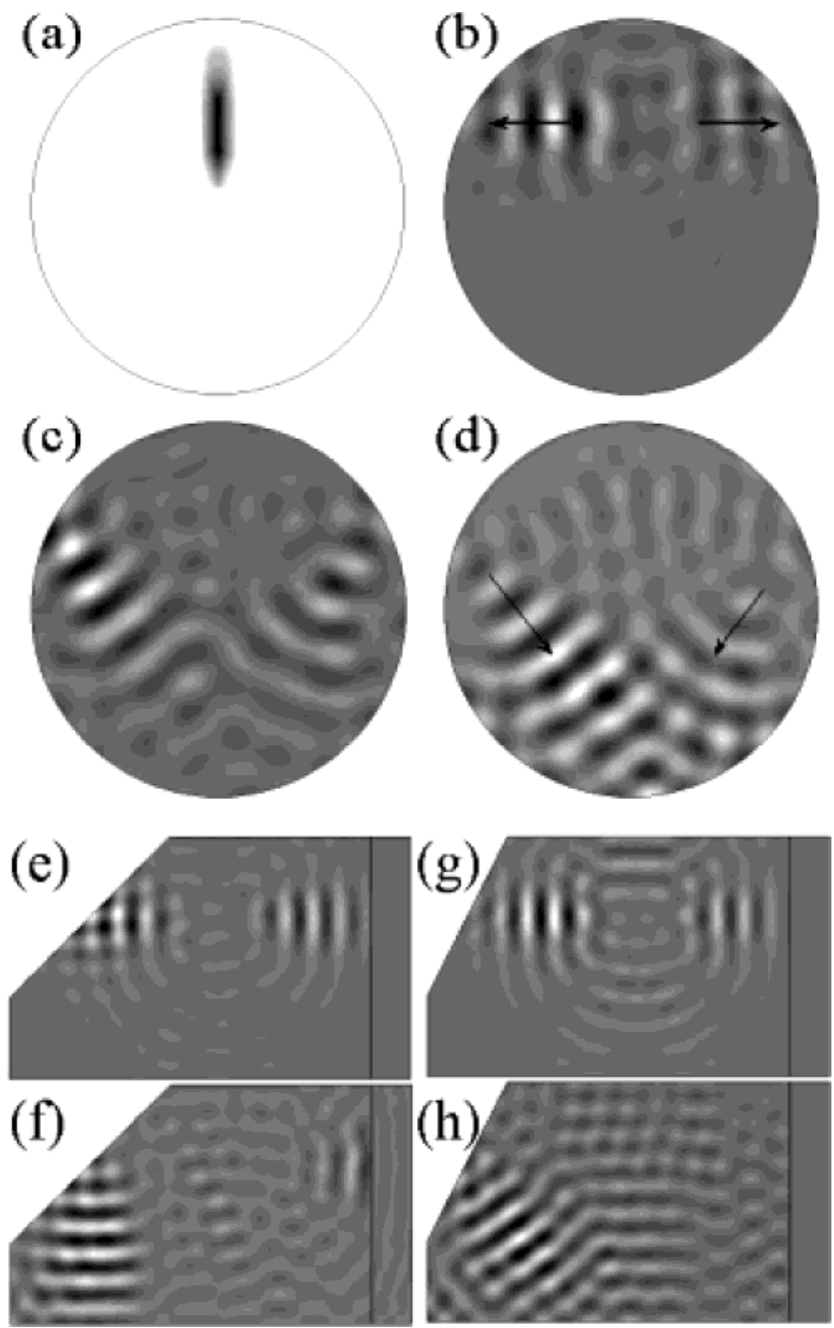

Figure 8. Reflection of packet waves. All parameters are as in Figure $1\left(\epsilon_{2}=0.61\right)$. Time after initial perturbation: $t=$ (a) 0.1 , (b) 8 , (c) 22 , (d) $32,(\mathrm{e}, \mathrm{g}) 12$, (f) 26 , (h) 34 . The radius of the circle $(\mathrm{a}-\mathrm{d})$ is 30. The total size of the rectangle $(\mathrm{e}-\mathrm{h})$ is $100 \times 60$, the damping rightsideband has width 10 (in this band, $\epsilon_{1}=0.025$, which gives negative $\operatorname{Re}(\lambda)$ for any wavenumber and prevents reflection from right boundary), and the excluded left top right triangle has sides 40 and 40 for (e, f) and 40 and 20 for $(\mathrm{g}, \mathrm{h})$. Zero-flux boundary conditions.

waves. To test this hypothesis, we performed computer experiments with a thin tube connected to a broader region (see Figure $9 \mathrm{c}, \mathrm{f}$ ). To our surprise, we found that passage of a wave packet through the mouth of the tube depends on the tube radius, $r$. We follow the wave packet by recording $u$ as a function of time at several spatial points (Figure 9a,b). At $t=L / V_{\mathrm{gr}}(\cong 12.8$ ), the center of the wave packet reaches the right end of the tube, as seen by the maximum in $u$ at $t=12$ in Figure $9 \mathrm{~b}(x=L)$. Then, the wave packet reflects from the right end of the tube and moves back toward the left tube end, resulting in the second maximum in Figure 9a $(x=L / 2)$ at $t \cong 22$. Reflection is not total, however. A small portion of the wave packet (measured as the ratio of the maximum amplitude of the transmitted waves in the rectangular area at $x=5 L / 4$ to the maximum amplitude of the incident wave packet in the tube at $x=3 L / 4$ ) passes through the tube end and enters the large rectangular area. The effect of the right tube end resembles that of a semitransparent mirror in a laser tube. The "coefficient of transparency" of this "mirror" depends on the tube radius, $r$. For large $r$, the major part of the wave packet enters the rectangular area (Figure 9f). The larger $r$, the larger the coefficient of transparency (Figure
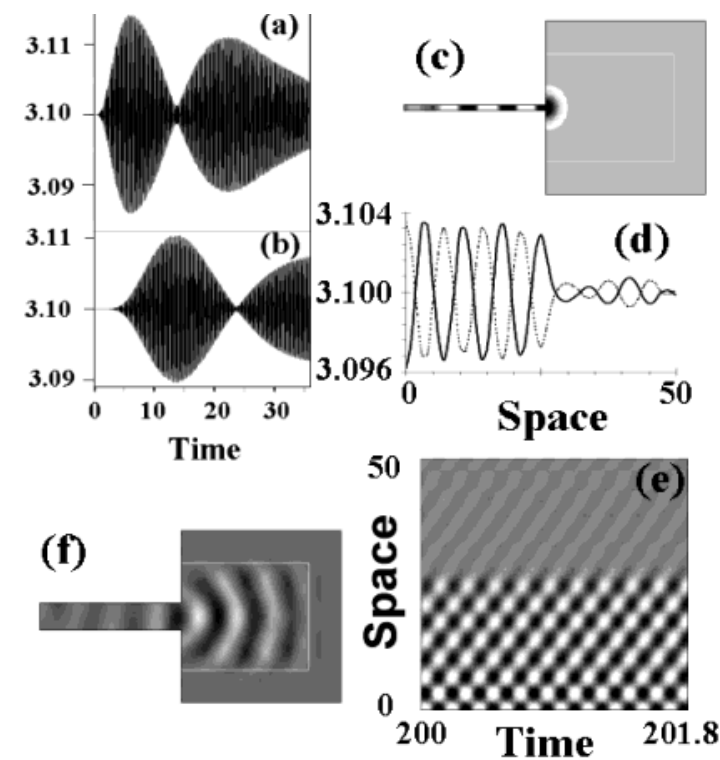

Figure 9. Packet waves in a tube with radius $r=0.5(\mathrm{a}-\mathrm{e})$ and $r=$ 2.5 (f). (a, b) Activator concentration $u$ at $x=$ (a) $L / 2$, (b) $L$. Length of

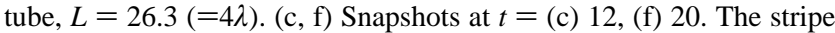
around the rectangular area of size $(50-L) \times 20$ has width $6 ; \epsilon_{1}=$ 0.025 in this stripe. (d) Profiles along the middle of the tube and rectangular area at $t=200.42$ and $t=200.51$ (half a period of oscillation). (e) Space-time plot, cross section along the middle of the tube and rectangular area; data accumulated with time interval 0.03. All parameters are as in Figure $1\left(\epsilon_{2}=0.61\right)$. Zero-flux boundary conditions.

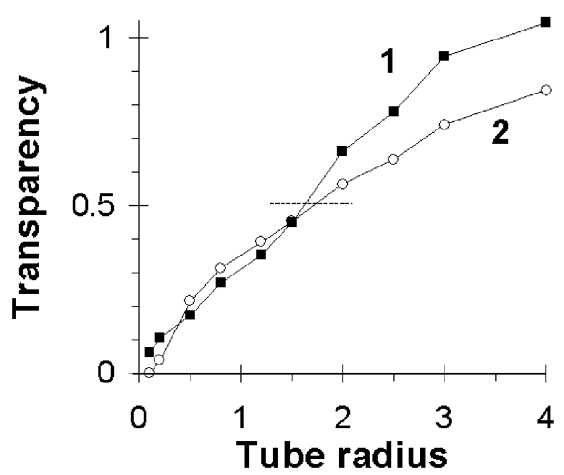

Figure 10. Dependence of coefficient of transparency on the tube radius determined by two different methods. (1) Ratio of the maximum amplitude of transmitted waves at $x=L+L / 4$ to a maximum amplitude of the wave packet in the tube at $x=L-L / 4$. (2) $1-$ the ratio of the maximum amplitude of reflected waves to the maximum amplitude of the incident wave packet at $x=L / 2$.

10). The critical radius, $r_{\mathrm{cr}}$, may be defined for packet waves as the tube radius at which the coefficient of transparency equals $50 \%$. With our parameters and with wave amplitudes measured at $x=L / 2$ and at $x=L \pm L / 4, r_{\text {cr }}=1.6-1.7$. Because the amplitudes of the reflected and transmitted waves may change with time in the active medium, the result is slightly dependent on the spatial points where the measurements of wave amplitude are made. For the same reason, the sum of the amplitudes of the reflected and transmitted waves is not exactly equal to the initial wave amplitude.

When $r<r_{\text {cr }}$, the wave packet reflects from the ends of the tube many times and a wave pattern similar to standing waves is established in the tube (Figure 9d,e) and continues to emit waves into the rectangular region. 


\section{Conclusion}

We have considered two limiting cases of wave behavior, pure packet waves and pure trigger waves, and have found new properties of each. Trigger waves can produce shock structures and exhibit acceleration of oncoming waves before collision. Packet waves undergo specular reflection at zero-flux boundaries. A "semitransparent mirror" can be constructed under special geometrical conditions for packet waves. Several questions remain. We still do not know why packet waves form spirals (when $\mathrm{d} \omega / \mathrm{d} k>0$ at $k=k_{0}$ ) or antispirals (when $\mathrm{d} \omega / \mathrm{d} k$ $<0$ at $k=k_{0}$ ), if eq 5 is not applicable to packet waves. What is the characteristic feature that transforms packet waves into trigger waves? What are the properties of hybrid trigger wavepacket wave patterns? At present, we can only report that, when $0.3<\epsilon_{2}<0.6$, complex wave behavior arises, with multiple wavelengths and internal reflection of waves of shorter wavelength from neighboring waves of longer wavelength within a single wave packet. Complexity increases as $\epsilon_{2}$ decreases, and chaotic behavior (in one spatial dimension, analyzed only by Fourier spectra at this stage) is found at about $\epsilon_{2}=0.4$.

Acknowledgment. We thank Lingfa Yang, Milos Dolnik, and Anatol M. Zhabotinsky for helpful discussions. This work was supported by the Chemistry Division of the National Science Foundation.

\section{References and Notes}

(1) Vanag, V. K.; Epstein, I. R. Phys. Rev. Lett. 2001, 87, 228301.

(2) Vanag, V. K.; Epstein, I. R. Science 2001, 294, 835-837.

(3) Vanag, V. K.; Epstein, I. R. Phys. Rev. Lett. 2002, 88, 088303

(4) Zhabotinsky, A. M.; Dolnik, M.; Epstein, I. R. J. Chem. Phys. 1995 103, 10306-10314.

(5) Hamik, C. T.; Manz, N.; Steinbock, O. J. Phys. Chem. A 2001, $105,6144-6153$.

(6) Kurin-Csörgei, K.; Zhabotinsky, A. M.; Orbán, M.; Epstein, I. R. J. Phys. Chem. A 1997, 101, 6827-6829. 657.

(7) Petrov, V.; Ouyang, Qi.; Swinney, H. L. Nature 1997, 388, 655-

(8) Perraud, J.-J.; De Wit, A.; Dulos, E.; De Kepper, P.; Dewel, G.; Borckmans, P. Phys. Rev. Lett. 1993, 71, 1272-1275.
(9) Osipov, V. V.; Muratov, C. B. Phys. Rev. Lett. 1995, 75, 338341.

(10) Vasilev, V. A.; Romanovskii, Ju. M.; Chernavskii, D. S.; Yakhno, V. G. Autowave Processes in Kinetic Systems. Spatial and Temporal SelfOrganization in Physics, Chemistry, Biology and Medicine; VEB Deutscher Verlag der Wissenschaften: Berlin, 1987.

(11) Vasilev, V. A.; Romanovsky, Ju. M.; Yakhno, V. G. Autowave Processes; Nauka: Moscow, 1987.

(12) Yang, L.; Epstein, I. R. J. Phys. Chem. A, submitted for publication.

(13) Nicolis, G.; Prigogine, I. Self-Organization in Nonequilibrium Systems; Wiley-Interscience: New York, 1977.

(14) FlexPDE. http://www.pdesolutions.com/flexpde.htm, 2001.

(15) Liboff, R. L. Introductory Quantum Mechanics; Addison-Wesley: Reading, MA, 1992.

(16) Loudon, R. In Order and Chaos in Nonlinear Physical Systems; Lundqvist, S., March, N. H., Tosi, M. P., Eds.; Plenum Press: New York, 1988; pp 225-252.

(17) Saxon, D. S. Elementary Quantum Mechanics; Holden-Day: San Francisco, 1968.

(18) Howard, L. N.; Kopell, N. Stud. Appl. Math. 1977, 56, 95145.

(19) Horikawa, Yo. Physica D 1995, 82, 365-370.

(20) Hamik, C. T.; Steinbock, O. Phys. Rev. E 2002, 65, 046224.

(21) Christoph, J.; Eiswirth, M.; Hartmann, N.; Imbihl, R.; Kevrekidis, I.; Bär, M. Phys. Rev. Lett. 1999, 82, 1586-1589.

(22) Elphick, C.; Meron, E.; Spiegel, E. A. Phys. Rev. Lett. 1988, 61, 496-499.

(23) Kerner, B. S.; Osipov, V. V. Autosolitons: A New Approach to Problems of Self-Organization and Turbulence; Kluwer: Dordrecht, The Netherlands. 1994.

(24) Merzhanov, A. G.; Rumanov, E. N. Rev. Mod. Phys. 1999, 71 $1173-1211$

(25) Nagumo, J.; Arimoto, S.; Yoshizawa, S. Proc. IRE 1962, 50, $2061-$ 2070.

(26) Tyson, J. J.; Fife, P. C. J. Chem. Phys. 1980, 73, 2224-2237.

(27) Winfree, A. T. Science 1972, 175, 634-636.

(28) Keener, J. P.; Tyson, J. J. Physica D 1986, 21, 307-324.

(29) Pertsov, A. M.; Wellner, M.; Jalife, J. Phys. Rev. Lett. 1997, 78 , $2656-2659$

(30) Zykov, V. S. Biophysics 1980, 25, 906-911.

(31) Tóth, A.; Gáspár, V.; Showalter, K. J. Phys. Chem. 1994, 98, 522531.

(32) Tóth, Á.; Showalter, K. J. Chem. Phys. 1995, 103, 20582066.

(33) Born, M. Principles of Optics; Pergamon: Oxford, U.K., 1970.

(34) Zhabotinsky, A. M.; Eager, M. D.; Epstein, I. R. Phys. Rev. Lett. 1993, 71, 1526-1529. 